\title{
Machine Learning Algorithms For Understanding The Determinants of Under-Five Mortality
}

\section{Rakesh Kumar Saroj ( $\nabla$ rakesh.saroj@bhu.ac.in )}

Sikkim Manipal Institute of Medical Sciences https://orcid.org/0000-0002-1651-2655

\section{Pawan Kumar Yadav}

International Institute for Population Sciences

\section{Rajneesh Singh}

Banasthali Vidyapith: Banasthali University

\section{Obvious Nchimunya Chilyabanyama}

Center for Infectious Disease Research in Zambia

\section{Methodology}

Keywords: decades, Random Forest, policymakers, breastfeeding

Posted Date: November 9th, 2021

DOI: https://doi.org/10.21203/rs.3.rs-1021040/v1

License: (c) (i) This work is licensed under a Creative Commons Attribution 4.0 International License. Read Full License 


\section{Abstract}

Background: The death rate of under-five children in India declined last few decades, but few bigger states have poor performance. This is a matter of serious concern for the child's health as well as social development. Nowadays, machine learning techniques play a crucial role in the smart health care system to capture the hidden factors and patterns of outcomes. In this paper, we used machine learning techniques to predict the important factors of under-five mortality.

This study aims to explore the importance of machine learning techniques to predict under-five mortality and to find the important factors that cause under-five mortality.

The data was taken from the National Family Health Survey-IV of Uttar Pradesh. We used four machine learning techniques like decision tree, support vector machine, random forest, and logistic regression to predict under-five mortality factors and model accuracy of each model. We have also used information gain to rank to know the important variables for accurate predictions in under-five mortality data.

Result: Random Forest (RF) predicts the child mortality factors with the highest accuracy of $97.5 \%$, and the number of living children, births in the last five years, educational level, birth order, total children ever born, currently breastfeeding, and size of child at birth that identifying as essential factors for under-five mortality.

Conclusion: The study focuses on machine learning techniques to predict and identify important factors for under-five mortality. The random forest model provides an excellent predictive result for estimating the risk factors of under-five mortality. Based on the resulting outcome, policymakers can make policies and plans to reduce under-five mortality.

\section{Introduction}

The most widely used measure is child mortality, for the health of children and an index of the general development of any country. Worldwide, under-five mortality rates are higher in South-Asian and Sub-Saharan African countries. The under-five mortality means is the probability of dying children before the fifth birthday. In India, the under-five child mortality rate is reduced by 49\%, from 83 deaths per 1000 live births in 2000 to 42 deaths in 2017 [1].

The state-wide reports have been found that under-five mortality is highest in Uttar Pradesh, followed by Madhya Pradesh and Chhattisgarh [2], as shown in Figure 1.

Although there has been a significant reduction in these state deaths of under-five children, it remains a major issue for child health. However, completing the Sustainable Goal-3 is still a challenging task for developing countries like India. The death rate of an under-five child can be prevented with early predictive analytics like machine learning techniques. Nowadays, machine learning techniques are highly demanded in public health research. The various machine learning models have been used for prediction and classification methods in different types of health and biomedical data. The machine learning approaches could help to obtain early prediction and insight into the important factors for under-five mortality. These all-ML models can automatically identify interactions and can find the nonlinear relationship between dependent and independent variables. There are various machine learning prediction and classification models like regression, logistic regression, principal component analysis, decision tree, and maximum likelihood method that have been used to find the accurate estimation of causes of child mortality.

Previous studies have used the traditional models for predicting the under-five mortality factors. The study suggests that a weak health system is one of the major problems of maximum under-five death of children in low middle-income countries [3].

The research study used the Cox proportional hazard $(\mathrm{CPH})$ model and frailty models to find under-five mortality risk factors [4]. The study has been applied classical and Bayesian approaches to the $\mathrm{CPH}$ model on under-five mortality data [5]. 
Another study has been done for finding the risk factors of under-five mortality with the help of the survival analysis method [6]. Within high-dimensional exposure data, machine learning (ML) approaches can be utilized to discover the exposures related to health outcomes of interest, as well as the potential interactions between those exposures [7]. The authors have been used the J48 and artificial neural network (ANN) techniques to find the causes of child mortality in Ethiopia [8]. The study was focused on assessing ML techniques' performance to predict the risk of neonatal mortality [9].

In the study, ML methods were used to predict neonatal morbidity and mortality [10]. ID3(Iterative Dichotomiser 3), random forest, and decision tree have been used for predicting the nutrition status in under-five age of children [11]. Another study was conducted in India to predict the nutrient effects on human health using ML techniques [12]. The research paper has been used a machine learning model to predict pre-term birth [13]. The above-cited research papers indicate that machine learning techniques have not been used in under-five mortality data. No single study is used for machine learning classification comparison in Indian and North Indian circumstances. Moreover, past studies have found a lack of a generic prediction framework for accurately estimating child mortality rates using machine learning techniques. There is a need for accurate prediction and classification models to provide highly accurate results and allow health researchers to experiment with various sets of aspects.

This study offers an opportunity to understand important factors and assess the accuracy of the machine learning techniques in under-five mortality. Apart from that, machine learning techniques explore the importance of predicting underfive mortality so that timely interventions can be made and factors that cause high rates of under-five mortality can be reduced.

\section{Methods}

This study has explained all the methods step by step through the proper framework for under-five mortality prediction. The explanations of the framework are portrayed in Figure 2. The detail of the methods explained.

\section{Dataset}

In the study, we have used secondary data from the National Family Health (NFHS-IV,2015-16) survey of Uttar Pradesh. NFHS-IV survey is a large-scale, multi-round survey conducted in households throughout the Indian states and union territories. The survey collects extensive information on population, health, and nutrition, with an emphasis on women and young children. We have used the ' 'kid's file from NFHS-IV of Uttar Pradesh. Total 41,751 sample is induvial with 1377 variables in this dataset.

\section{Data Pre-processing}

Pre-processing required cleaning the data, removing irrelevant or duplicate records, and handling missing values, for making it suitable to fit in a machine learning model, which helps to increase the accuracy and efficiency of the model. This is the step in which data gets transformed or encoded to bring it to a state such that the machine learning algorithms can easily interpret it. After all, did we have selected 18 variables and 41,751 individuals from the dataset for final ML analysis

\section{Feature ranking using information gain}

Feature ranking using information gain gives the idea about the most important factors from the dataset through ranking. This is based on the calculation of the information gain values for each of the selected variables. In this study, we have used a random forest model for finding the risk factors or important features that are majorly contributing to the mortality of children. The higher values of information gain tell that are important variables and highly correlated with the class variable. Finally, the top eight ranked information values are used in the model building later.

\section{Model building}




\section{Data Splitting}

In this step, we split the datasets into trained and test. $70 \%$ of the trained data are used for the ' 'model's classification and $30 \%$ data for model evaluation. The train data should be greater than test data for avoiding over-fitting and under-fitting and increasing the efficiency of the model. Then the outcome variable or dependent feature needs to be transformed into binary form by one-zero encoding, and independent features can be continuous or categorical and need to be in one data type format. We have used the important machine learning models like decision tree, random forest, support vector, and logistic regression in trained datasets.

\section{Model Evaluation}

To assess each model's effectiveness in the $30 \%$ test data. This procedure helps us to ensure which model performed the best for predicting child mortality. There is various way to measure the accuracy of the model; details are given in Figure 3 .

The confusion matrix is a table that is often used to describe the performance of a classification model in the set of test data. The table is like a contingency table, with two dimensions actual and predicted value. These are the following concept is defined in the confusion matrix table.

True positive (TP) - The model correctly predicts positive class in the outcome.

True negative (TN) - The model correctly predicts negative class in the outcome.

False-positive (FP) - The model incorrectly predicts positive class in the outcome.

False-negative (FN) - The model incorrectly predicts negative class in the outcome.

Additionally, we have used accuracy, precision, recall, and F1-score for the performance of the model of the test data.

\section{Results}

\section{Risk Factors Analysis}

We have developed the all-classification model for prediction in training data (70\%) with all factors. The models were tested in test data (30\%) and used the confusion matrix table to describe the performance of a classification model in the set of test data. The result of the confusion matrix table is explained in Figure 4. The result of the model evaluation is explained in Table 1, and the highest accuracy is the random forest model with $97.5 \%$ compared with other models.

Table 1

Performance of ML models with all factors

\begin{tabular}{|c|c|c|c|c|c|c|c|c|c|c|c|c|}
\hline \multirow[t]{2}{*}{ Measure } & \multirow[t]{2}{*}{ DT } & \multicolumn{2}{|c|}{ 95\% C.I. } & \multirow[t]{2}{*}{ SVM } & \multicolumn{2}{|c|}{ 95\% C.I. } & \multirow[t]{2}{*}{ RF } & \multicolumn{2}{|c|}{ 95\% C.I. } & \multirow[t]{2}{*}{ LR } & \multicolumn{2}{|c|}{ 95\% C.I. } \\
\hline & & Lower & Upper & & Lower & Upper & & Lower & Upper & & Lower & Upper \\
\hline Recall & 0.581 & 0.546 & 0.616 & 0.502 & 0.467 & 0.537 & 0.743 & 0.711 & 0.773 & 0.521 & 0.485 & 0.556 \\
\hline Precision & 0.861 & 0.830 & 0.886 & 0.800 & 0.765 & 0.831 & 0.872 & 0.846 & 0.894 & 0.767 & 0.732 & 0.799 \\
\hline Accuracy & 0.965 & 0.962 & 0.968 & 0.958 & 0.954 & 0.961 & 0.975 & 0.972 & 0.978 & 0.957 & 0.953 & 0.96 \\
\hline F1 Score & 0.694 & 0.671 & 0.717 & 0.617 & 0.592 & 0.641 & 0.802 & 0.782 & 0.823 & 0.620 & 0.595 & 0.645 \\
\hline
\end{tabular}


Thereafter, we have used a random forest model for finding the risk factors or important features that are majorly contributing to the mortality of children. We use the random forest to check feature importance concerning its predictive power.

We drop all factors whose rank value is less than 0.07 because the accuracy of the model will justify this level only. The result of feature importance explains in Figure 5. The result shows that the most important determinants of under-five child mortality are the number of living children, birth in the last five years, education level, birth order, and child size at birth because they are high rank in order. Other determinants like total children ever born; currently, breastfeeding and exposure are low ranks in order means they have less relation with child mortality.

Later we have repeated all procedures once again with important ranking factors to know the importance of information gain measure or very important features (VIMP). The details of the confusion matrix explained in Figure 6, and the result of model evaluation is given in Table 2. Again, the highest accurate model is the RF model, with $97 \%$ accuracy compared to other models. It is found that nearly the same accuracy value is showing in all the models. The analysis tells that number of living children, birth in the last five years, education level, birth order, and child size at birth are important factors in under-five mortality. However, for performing the prediction of the model, other factors also play a vigorous role, but major factors are the same for under-five mortality.

Table 2

Performance of ML models with important factors

\begin{tabular}{|c|c|c|c|c|c|c|c|c|c|c|c|c|}
\hline \multirow[t]{2}{*}{ Measure } & \multirow[t]{2}{*}{ DT } & \multicolumn{2}{|c|}{ 95\% C.I. } & \multirow[t]{2}{*}{ SVM } & \multicolumn{2}{|c|}{ 95\% C.I. } & \multirow[t]{2}{*}{ RF } & \multicolumn{2}{|c|}{ 95\% C.I. } & \multirow[t]{2}{*}{ LR } & \multicolumn{2}{|c|}{ 95\% C.I. } \\
\hline & & Lower & Upper & & Lower & Upper & & Lower & Upper & & Lower & Upper \\
\hline Recall & 0.525 & 0.489 & 0.560 & 0.502 & 0.467 & 0.537 & 0.681 & 0.648 & 0.714 & 0.523 & 0.488 & 0.558 \\
\hline Precision & 0.768 & 0.734 & 0.800 & 0.800 & 0.765 & 0.831 & 0.846 & 0.818 & 0.870 & 0.765 & 0.730 & 0.797 \\
\hline Accuracy & 0.957 & 0.953 & 0.961 & 0.958 & 0.954 & 0.961 & 0.970 & 0.967 & 0.973 & 0.957 & 0.953 & 0.960 \\
\hline F1 Score & 0.623 & 0.599 & 0.648 & 0.617 & 0.592 & 0.641 & 0.755 & 0.733 & 0.776 & 0.622 & 0.598 & 0.647 \\
\hline
\end{tabular}

Overall result highlights that the predictors of under-five child mortality and information gain ranked (VIMP) that factors are highly associated with under-five child mortality. It is also showed that the RF classifier model is the most accurate model for predicting the under-five child mortality in the predictive analytics structure. This research shows the importance of a machine learning framework in health data. With this framework, scientists, health researchers, epidemiologists, and applied researchers can predict and find the important factors of outcome in health data. Finally, this research tells the important factors which are responsible for under-five mortality. Based on the research work on those factors to reduce under-five mortality.

\section{Discussion}

This study evaluated the importance of machine learning techniques in predicting the factors of under-five mortality. The kinds of literature say that this is the first study that used machine learning techniques in the high mortality data of India Uttar Pradesh for predicting the under-five mortality. Regarding the predictive analysis, the prediction accuracies are found to be highest (97.5\%) in the RF model. The RF model also shows that the number of living children, birth in the last five years, education level, birth order, child-size at birth, total children ever born, currently breastfeeding, and exposure are the top eight important predictors of under-five mortality. Various studies also confirm that these factors are crucial for under-five mortality [14-18]. 
The finding of the result shows only a smaller number of factors of under-five child mortality comparison between ML model and traditional models like logistic regression and survival analysis. This is an indication of the importance of the ML model in under-five mortality studies. This research finds that the mother's education level is one of the major factors of under-five mortality through the ML model and the previous result also confirms the same result [19]. The study has found that birth lasts five years, and birth order is an important factor against under-five mortality. These results are similar to the study reported using the ML approach [20] and traditional methods [21]. As per this study, prior research has also found that baby size at birth and breastfeeding were significant determinants of under-five mortality [22]. The study has used variables like mother literacy, a birth rate affecting child mortality, and the random-forest model finds the best-suited model with high accuracy [23]. The study shows the importance of machine learning for predicting the risk factors of infant mortality in India [24] and thus needs a proper plan to reduce the infant mortality rate. The research article used the machine learning predictive model to predict the risk of neonatal death and stillbirth in low middle-income countries [25]. This study has measured the various child health indicators, including under-five mortality of India, through machine learning techniques [26]. However, ML models are considered to be very valuable in predicting health studies and lead to healthier and suitable policy decisions [27].

This study idea cannot complete without its limitation because we are using the machine learning models, unlikely statistical models. The ML model's result comes without any coefficient and odds ratio compared to the statistical model then it's unpredicted to understand how much and which direction factors affect the outcome. We have to decide our research hypothesis in the study because ML models can be used for prediction and classification both manners. The result of the study is based on the NFHS questionnaires data, not a specific study or objective related to under-five mortality.

\section{Conclusion}

The child mortality of under the age of five is one of the major causes of child survival in India, especially in bigger states like Uttar Pradesh. This study explains the importance of ML models in predicting the important factors and understanding under-five mortality. The random forest $(\mathrm{RF})$ model gives the better and accurate predictive power in this study. This model provides some important factors that may not be able to get by other traditional statistical models. These findings emphasize the need to focus on the most significant predicted factors, including breastfeeding, birth interval control, and antenatal care, in developing policies for reducing under-five mortality. The model can be helpful for better policy directions regarding under-five child survival. The finding of the study indicates that the ML model may apply to find the risk factors and produce higher accuracy.

\section{Declarations}

\section{Authors' contributions}

RKS developed the concept of paper, analysed the data, makes an algorithm for model evaluation and comparison. Write the maximum part of the manuscript. PKS helps in the writing of the manuscript and cleanses the data and makes appropriate for analysis.RS and ONC help with the construction of manuscript, help in the coding and gives the accurate comment for revising the manuscript. All the author's read and approved the final paper.

\section{Availability of data and materials}

The data of the National Family Health Survey is available online. The International Institute for Population Sciences (IIPS), Mumbai website is the nodal agency for the NFHS-4 survey. This data is freely available to access for research to anyone after registration.

\section{Ethics approval and consent to participate}


This study was used to analysed a secondary data set, and it has no identifiable information of the survey participants. This dataset is easily available in the public domain for research purposes; hence no approval was required from any institutional review board as there is no question of human subject protection arises in this case.

\section{Consent for publication}

Not applicable

\section{Competing interests}

The authors declared that they have no competing interests

\section{Author Details}

${ }^{1}$ Department of Community Medicine, Medicine, Sikkim Manipal Institute of Medical Sciences-Gangtok-737102, India. ${ }^{2}$ Department of Development Studies, International Institute for Population Sciences, Mumbai-India. ${ }^{3}$ Department of Mathematics and Statistics, Banastahli Vidya Pith, Rajasthan-India. ${ }^{4}$ Centre for Infectious Disease Research in Zambia, Lusaka, Zambia.

\section{References}

1. IIPS, ICF. National Family Health Survey (NFHS-4), 2015-16: India. Mumbai: International Institute for Population Sciences 2017.

2. http://rchiips.org/nfhs/NFHS-4Reports/India.pdf (access on 23/07/2021 at 2.50 PM (IST)).

3. Kruk ME, Porignon D, Rockers PC, Van Lerberghe W. The contribution of primary care to health and health systems in low-and middle-income countries: a critical review of major primary care initiatives. Social science \& medicine. 2010;70(6):904-11.

4. Ayele DG, Zewotir TT, Mwambi H. Survival analysis of under-five mortality using Cox and frailty models in Ethiopia. Journal of Health, Population and Nutrition. 2017 ;36(1):1-9.

5. Nilima S. Under-five child mortality in Bangladesh: Classical and Bayesian approaches to Cox proportional hazard model. Bangladesh Journal of Scientific Research. 2017;30(1-2):45-54.

6. Saroj RK, Murthy KN, Kumar M. Survival analysis for under-five child mortality in Uttar Pradesh. Int J Res Anal Rev. 2018 ;59:782-9.

7. Patel CJ. Analytic complexity and challenges in identifying mixtures of exposures associated with phenotypes in the exposome era. Current epidemiology reports. 2017 ;4(1):22-30.

8. Tesfaye B, Atique S, Elias N, Dibaba L, Shabbir SA, Kebede M. Determinants and development of a web-based child mortality prediction model in resource-limited settings: a data mining approach. Computer methods and programs in biomedicine. $2017 ; 140: 45-51$.

9. Alves LC, Beluzo CE, Arruda NM, Bressan R, Carvalho T. Assessing the Performance of Machine Learning Models to Predict Neonatal Mortality Risk in Brazil, 2000-2016. medRxiv. 2020.

10. Jaskari J, Myllärinen J, Leskinen M, Rad AB, Hollmén J, Andersson S, Särkkä S. Machine learning methods for neonatal mortality and morbidity classification. IEEE Access. 2020 ;8:123347-58.

11. Thangamani D, Sudha P. Identification of malnutrition with use of supervised datamining techniques-decision trees and artificial neural networks. Int J Eng Comput Sci. $2014 ; 3(09)$.

12. Kuttiyapillai $D$, Ramachandran R. Improved text analysis approach for predicting effects of nutrient on human health using machine learning techniques. IOSR J Comput Eng. 2014;16(3):86-91. 
13. Raja R, Mukherjee I, Sarkar BK. A Machine Learning-Based Prediction Model for Preterm Birth in Rural India. Journal of Healthcare Engineering. 2021;2021.

14. Majumder AK, May M, Pant PD. Infant and child mortality determinants in Bangladesh: Are they changing?. Journal of Biosocial Science. 1997 ;29(4):385-99.

15. Hong R, Hor D. Factors associated with the decline of under-five mortality in Cambodia, 2000-2010: Further analysis of the Cambodia Demographic and Health Surveys. Calverton: ICF International. s. 2013.

16. Dendup T, Zhao Y, Dema D. Factors associated with under-five mortality in Bhutan: an analysis of the Bhutan National Health Survey 2012. BMC public health. 2018;18(1):1-5.

17. Saroj RK, Murthy KH, Kumar M, Singh R, Kumar A. Survival parametric models to estimate the factors of under-five child mortality. Journal of Health Research and Reviews. $2019 ; 6(2): 82$.

18. Islam M, Usman M, Mahmood A, Abbasi AA, Song OY. Predictive analytics framework for accurate estimation of child mortality rates for Internet of Things enabled smart healthcare systems. International Journal of Distributed Sensor Networks. $2020 ; 16(5): 1550147720928897$.

19. Bitew FH, Nyarko SH, Potter L, Sparks CS. Machine learning approach for predicting under-five mortality determinants in Ethiopia: evidence from the 2016 Ethiopian Demographic and Health Survey. Genus. 2020;76(1):1-6.

20. Panesar SS, D'Souza RN, Yeh FC, Fernandez-Miranda JC. Machine learning versus logistic regression methods for 2year mortality prognostication in a small, heterogeneous glioma database. World neurosurgery: X. 2019 Apr 1;2:100012.

21. Mani K, Dwivedi SN, Pandey RM. Determinants of under-five mortality in Rural Empowered Action Group States in India: An application of Cox frailty model. International Journal of MCH and AIDS. 2012;1(1):60.

22. Holzinger A. Introduction to MAchine Learning \& Knowledge Extraction (MAKE). Mach. Learn. Knowl. Extr.. 2019;1(1):120.

23. Gawande R, Indulkar S, Keswani H, Khatri M, Saindane P. Analysis and Prediction of Child Mortality in India. International Research Journal of Engineering and Technology. 2019;6(3):5071-4.

24. Singha AK, Phukan D, Bhasin S, Santhanam R. Application of machine learning in analysis of infant mortality and its factors. Work Pap. 2016:1-5.

25. Shukla VV, Eggleston B, Ambalavanan N, McClure EM, Mwenechanya M, Chomba E, Bose C, Bauserman M, Tshefu A, Goudar SS, Derman RJ. Predictive modeling for perinatal mortality in resource-limited settings. JAMA network open. $2020 ; 3(11)$ :e2026750-.

26. Bhattacharjee B. Child Health in India: An Application of Machine Learning. Turkish Journal of Computer and Mathematics Education (TURCOMAT).2021 24;12(8):2122-7.

27. Ashrafian H, Darzi A. Transforming health policy through machine learning. PLoS Medicine. 2018;15(11):e1002692.

\section{Figures}




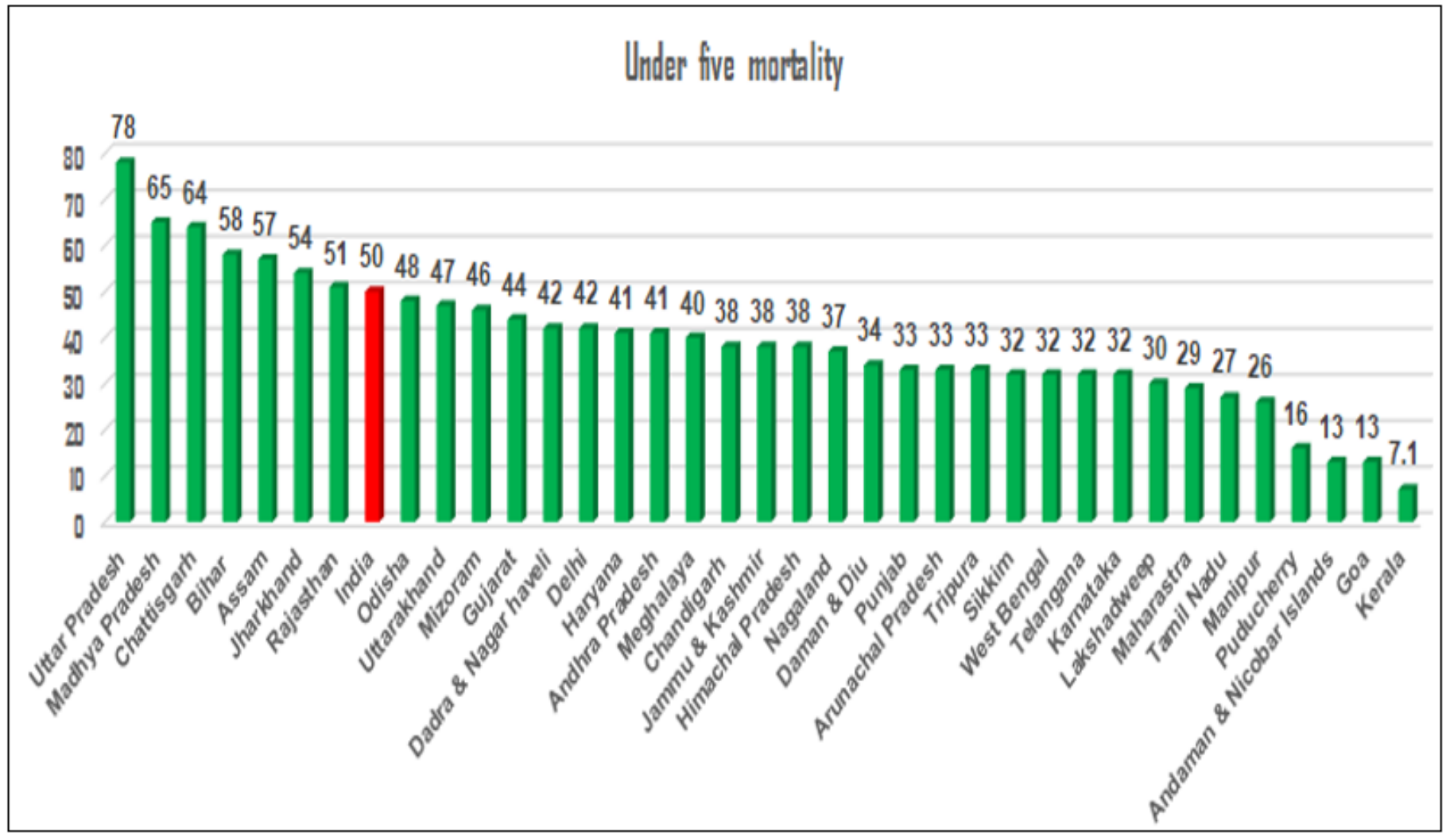

Figure 1

Under-five mortality of Uttar Pradesh comparison graph of State-Wise-India (NFHS-4) 


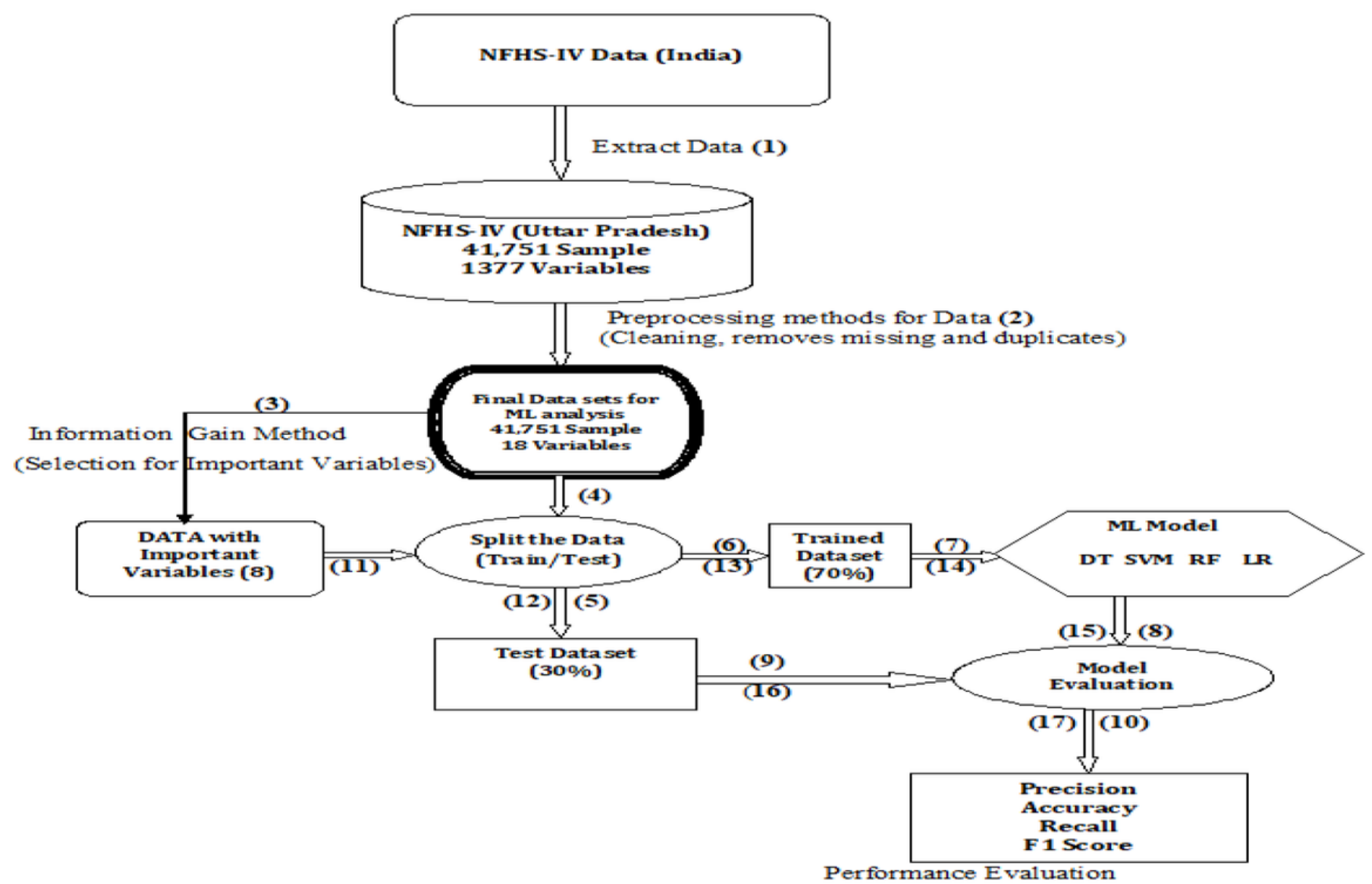

Figure 2

Overview of the proposed framework for under-five child mortality

\begin{tabular}{|c|c|c|c|}
\hline & & \multicolumn{2}{|c|}{ Predicted } \\
\hline \multirow{4}{*}{ Observed } & & Positive (Live) & Negative (Death) \\
\cline { 2 - 4 } & Positive (Live) & TP & FN \\
\cline { 2 - 4 } & Negative (Death) & FP & TN \\
\hline
\end{tabular}

\begin{tabular}{l|l||}
\hline Measure & Formula \\
\hline Recall & $\mathrm{TPR}=\mathrm{TP} /(\mathrm{TP}+\mathrm{FN})$ \\
\hline Precision & $\mathrm{PPV}=\mathrm{TP} /(\mathrm{TP}+\mathrm{FP})$ \\
\hline Accuracy & $\mathrm{ACC}=(\mathrm{TP}+\mathrm{TN}) /(\mathrm{TP}+\mathrm{TN}+\mathrm{FP}+\mathrm{FN})$ \\
\hline F1 Score & $\mathrm{F} 1=2 \mathrm{TP} /(2 \mathrm{TP}+\mathrm{FP}+\mathrm{FN})$ \\
\hline
\end{tabular}

Figure 3

Confusion matrix and model evaluation measure features 

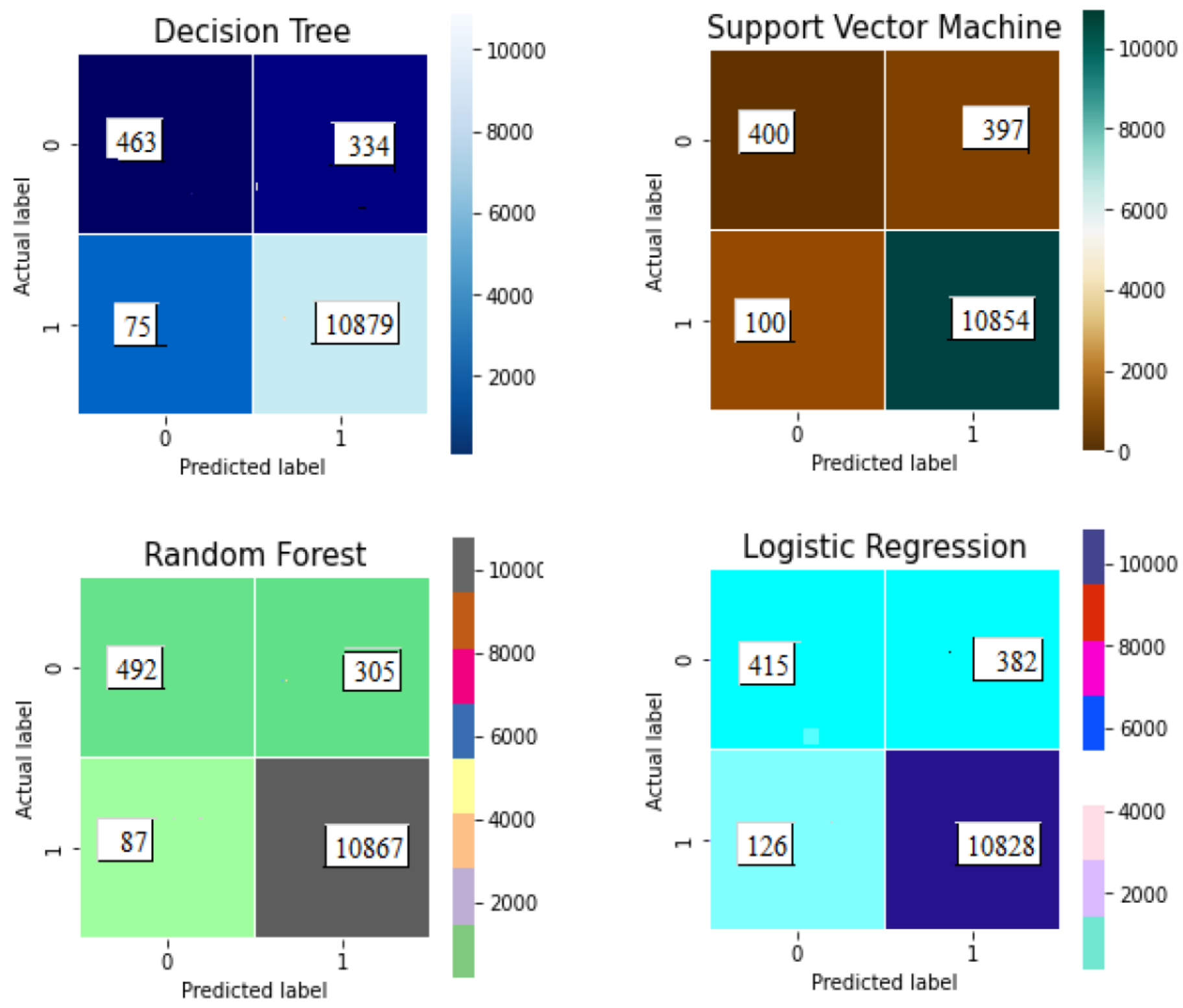

Figure 4

Confusion matrix with all factors 


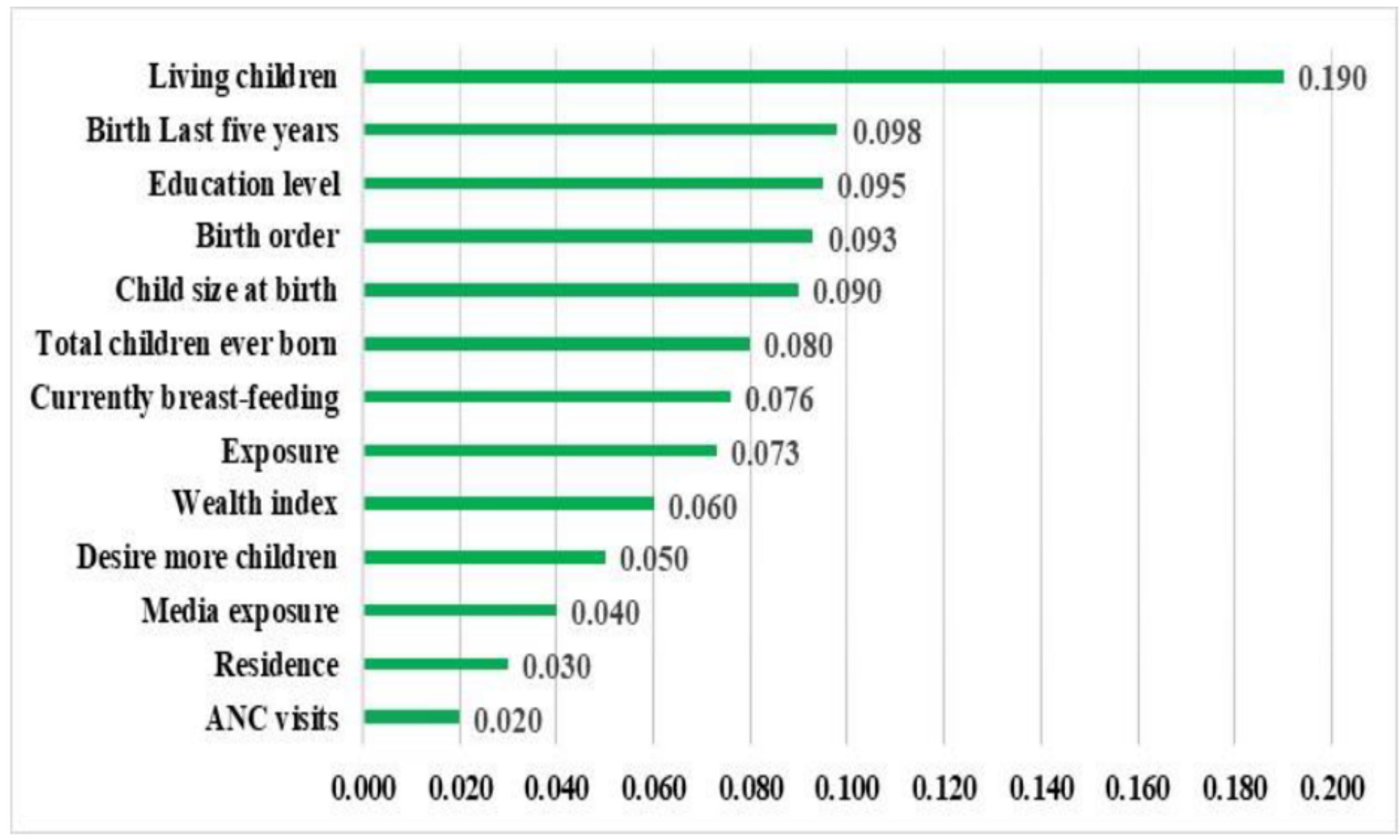

Figure 5

Information gain rank values of the variables 

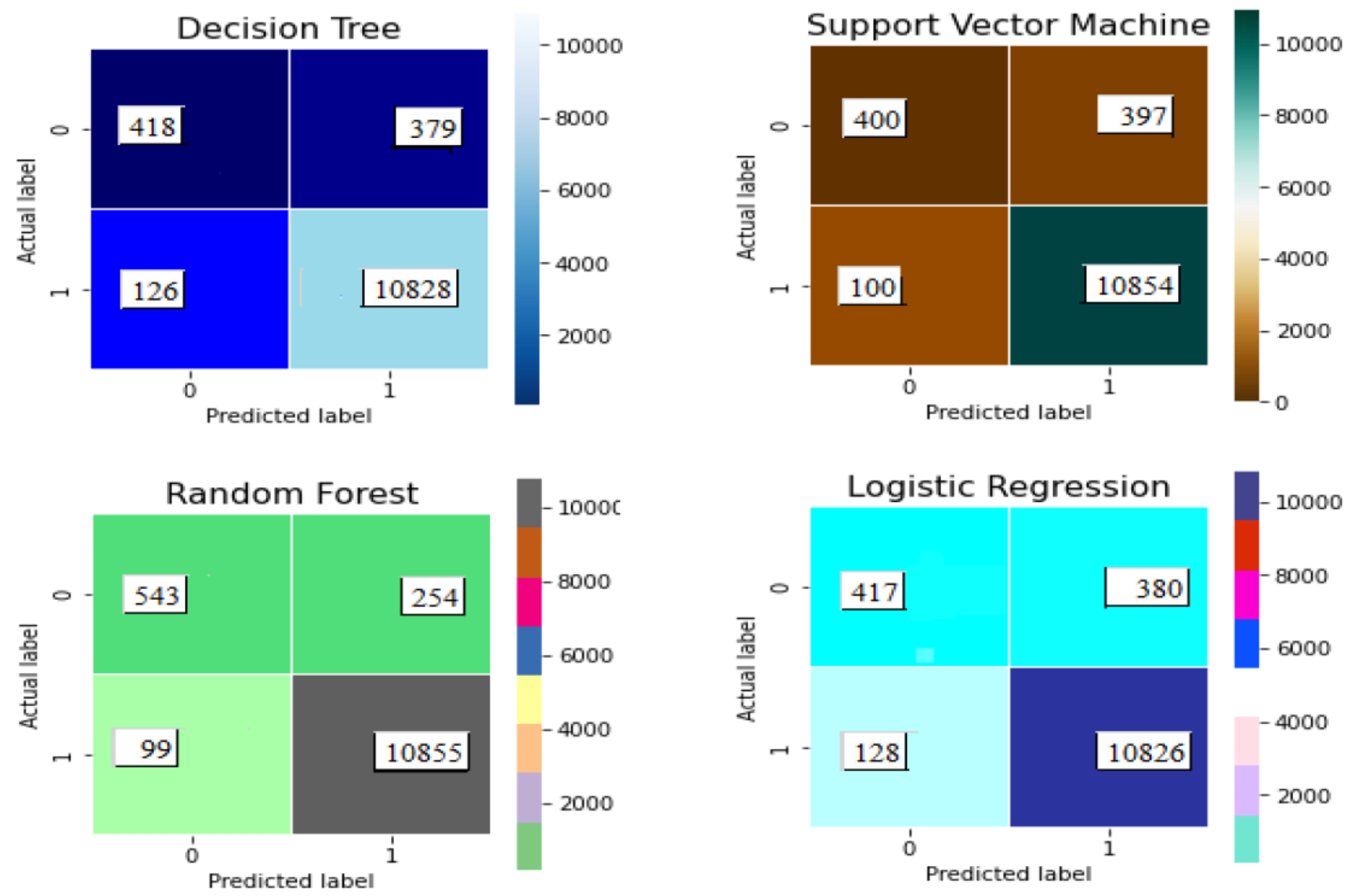

Figure 6

Confusion matrix with important factors 